\title{
DLBCL pelvic lymphoma - non-Hodgkin's lymphoma. Application of the US I MR imaging method in diagnosis and treatment monitoring.
}

\author{
Authors: Maria Kowalczuk ${ }^{1}$, Justyna Lipińska ${ }^{1}$, Marek Kurowski ${ }^{1}$, Magdalena Woźniak ${ }^{2}$ (mentor) \\ ${ }^{1}$ Students' Scientific Society at the Department of Pediatric Radiology, Lublin, Poland \\ ${ }^{2}$ Department of Pediatric Radiology, Lublin, Poland \\ DOI: https://doi.org/10.26800/LV-142-supp5-12
}

\section{Background:}

Lymphomas account for about $2 \%$ of all malignant neoplasms. They are neoplastic diseases characterized by massive growth of cells in the lymphatic system. Diffuse large B-cell lymphomas (DLBCL) are the most common subtype. DLBCL is derived from mature, peripheral $B$ lymphocytes of germination. Lymph nodes are the most common localization, but lymphoma is also primarily located in extra-nodal structures: stomach, tonsils, spleen, thymus, skin, thyroid gland, bones and brain.

\section{Case presentation:}

A 17-year-old patient with lower abdominal pain was admitted to the University Children's Hospital in Lublin, where in August 2020, pelvic MRI with contrast was performed for diagnosis. Within the pelvis, a pathological, irregular tissue masses with fluid areas were visualized. Additionally on the left side of the pelvis, in the area of the described lesions, as well as within the abdominal cavity, numerous lowsignal areas of 6-27 $\mathrm{mm}$ in size: probably pathological lymph nodes were visible. The described changes after contrast administration were enhanced and showed features of restriction in DWI images. On the basis of histopathological examination, the diagnosis of non-Hodgkin's lymphoma from large B lymphocytes in the small pelvis was confirmed. Adequate chemotherapy was implemented.

In the follow-up US examination performed in September, a tissue mass in the smaller pelvis of much smaller dimensions than the original was visualized. The Color Doppler option did not detect any obvious vascularity as a feature of the response to the applied treatment.

\section{Conclusion:}

In summary, both MRI and ultrasound are valuable imaging modalities for the diagnosis and treatment monitoring of DLBCL lymphoma. However, it should be remembered that the histopathological examination is of decisive importance in the differential diagnosis of lymphomas.

Keywords: Iymphoma, neoplasm, pelvic lymphoma, MRI 